Randomized Trial

\title{
Comparison of Epidural Spreading Patterns and Clinical Outcomes of Transforaminal Epidural Steroid Injection with High-Volume Injectate via the Subpedicular Versus the Retrodiscal Approach
}

Won-Joong Kim, MD, PhD'1, Hwa-Yong Shin, MD, PhD², Seung Hee Yoo, MD', and Hahck Soo Park, MD, PhD ${ }^{1}$

From: ${ }^{1}$ Department of Anesthesiology and Pain Medicine, School of Medicine,

Ewha Womans University

Mokdong Hospital, Seoul, Republic of Korea; ${ }^{2}$ Department of Anesthesiology and Pain Medicine, Chung-Ang University College of Medicine, Dongjakgu, Seoul, Republic of Korea

Address Correspondence: Hahck Soo Park, MD, PhD Associate Professor

Department of Anesthesiology and Pain Medicine

School of Medicine, Ewha

Womans University

1071 Anyangcheon-ro, Yangcheon-gu

Seoul, Republic of Korea

E-mail: ingoo97@naver.com

Disclaimer: There was no

external funding in the preparation of this manuscript. Conflict of interest: Each author

certifies that he or she, or a member of his or her immediate

family, has no commercial association (i.e., consultancies, stock ownership, equity interest, patent/licensing arrangements, etc.) that might pose a conflict of interest in connection with the submitted manuscript.

Manuscript received: 07-21-2017 Revised manuscript received: 10-26-2017

Accepted for publication: 11-17-2017

Free full manuscript: www.painphysicianjournal.com
Background: The effect of transforaminal epidural steroid injection (TFESI) appears to be influenced by the volume of the injected material because there is a positive correlation between injection volume and extent of pain relief.

Objectives: The purpose of this study was to examine how many vertebral segments are covered by the subpedicular (SP) approach and the retrodiscal (RD) approach and to compare the clinical outcomes of the 2 approaches in TFESI with high-volume injectates.

Study Design: A randomized, active control trial.

Setting: Medical University centers.

Methods: Fifty patients were randomly assigned to either the SP group or the RD group. TFESI was performed with high-volume injectates. A total of $9 \mathrm{~mL}$ injectate was divided into 3 injections $(0.5 \mathrm{~mL}, 2.5 \mathrm{~mL}$, and $6 \mathrm{~mL})$ given at 10 -second intervals. The primary outcome measure was injectate distribution. The spreading patterns were described as unilateral, bilateral, ventral, or dorsal. Ventral or dorsal flow was also described as being cephalad or caudad, respectively. The secondary outcome measures were pain relief and reduction of functional disability at 4 weeks after the procedure.

Results: The total numbers of vertebral segments and median levels of contrast spread from the injection site were not significantly different between the 2 groups. However, in $3 \mathrm{~mL}$ of injectate, the injectate spread showed more extensive bilateral distribution in the RD group. At 4 weeks after treatment, both groups demonstrated statistically significant pain relief and improvement in functional status. No significant differences were observed between the 2 groups.

Limitations: We enrolled only a small number of patients and did not analyze long-term outcomes. Patients with severe spinal stenosis or herniated nucleus pulposus were included Slightly different methods were used in the 2 groups to determine the number of levels at which the injectate was spread.

Conclusions: Epidural spreading patterns and clinical outcomes of lumbar TFESI with highvolume injectate were not significantly different between the SP approach and the RD approach.

Institutional Review Board (IRB) approval: Institutional Review Board of Ewha Womans University Hospital (EUMC 2015-05-003-003). Clinical trials registration number: Clinical Research Information Service (CRIS, registration number: KCT0002288; RCT URL: https://cris.nih.go.kr/cris/ search/search_result_st01.jsp?seq=7309)

Key words: Epidural injections, epidural space, low back pain, radiating pain, disability evaluation, steroids, local anesthetics, intervertebral disc disease, spinal stenosis

Pain Physician 2018; 21:269-278 
A $\mathrm{n}$ epidural steroid injection (ESI) procedure can take an interlaminar, caudal, or transforaminal (TF) approach, depending on the particular site at which a needle is inserted into the epidural space. The TF approach is generally preferred because delivery of a therapeutic injectate transforaminally at the involved nerve root maximizes steroid concentration at the site of pathology; moreover, the injectate can be delivered to the anterior epidural space of the symptom-associated nerve root $(1,2)$.

Several mechanisms have been suggested to explain the anti-inflammatory action of epidural corticosteroids on lumbosacral radiculopathy $(3,4)$. These corticosteroids can inhibit the production of arachidonic acid or the occurrence of ectopic discharge from unmyelinated C-fibers. Alternatively, they can relieve central sensitization $(5,6)$. The effect of TFESI might be influenced by the volume of the injected material. A positive correlation has been reported between fluid injection volume into the epidural space and relief of radicular leg pain and low back pain (7). The injected material displaces the dura forward and inward, in addition to stretching the nerve roots. These effects lead to lysis of neural adhesions (7).

The TF approach can be divided into 3 approaches - the subpedicular (SP) approach, the retroneural (RN) approach, and the retrodiscal (RD) approach - depending on where the needle is advanced (Fig. 1). The SP ap- proach is currently used most frequently in clinical practice. In this approach, an injection needle is advanced into the safe triangle under the inferior aspect of the pedicle, as described by Bogduk (8). Two alternative approaches for TFESI include the RN approach and the RD approach. The RN approach has an optimal target area more dorsal to the intervertebral foramen compared with the SP approach (9). In the RD approach, the needle is advanced into the inferior aspect of the foramen (10). This technique has the advantage that the injection can be made closest to the nerve root, which is stimulated by a prolapsed intervertebral disc (IVD). However, it is not clear which TFESI method is preferred for treatment of lumbar radiculopathy. Some studies have concluded that TFESI for lumbar radiculopathy with the RD approach is more effective than TFESI with the SP approach (11), but others have reached different conclusions (12).

To the best of our knowledge, no controlled trials have examined the epidural spreading patterns or clinical outcomes of a high-volume injectate via the SP approach versus the RD approach in patients undergoing lumbar TFESI. The purpose of this study was therefore to determine how many spinal segments are covered by the two approaches and to compare the clinical effects of delivering a high-volume injectate (with the same dose of dexamethasone) by the 2 approaches.
$\mathbf{A}$

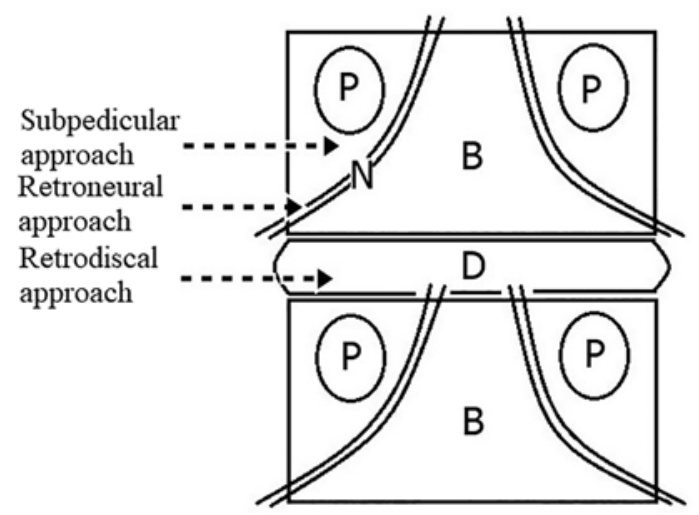

B

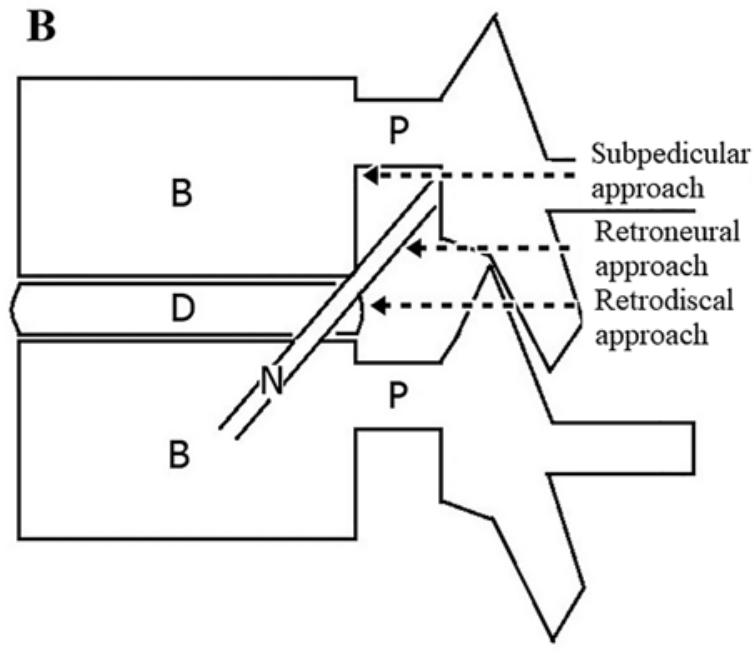

Fig. 1. Three transforaminal approaches. (A) Anteroposterior view (B) Lateral view.

B: vertebral body, D: intervertebral disc, N: spinal nerve, P: pedicle 


\section{MethODS}

This randomized, active control trial study was conducted with the full approval of the Institutional Review Board of Ewha Womans University Hospital (EUMC 2015-05-003-003) and in accordance with the ethical principles of the Declaration of Helsinki. An informed consent was presented to all patients prior to enrollment in the study groups. Written informed consent to participate were obtained from all the patients.

\section{Randomization}

Fifty patients were enrolled and randomly assigned to either the SP group or the RD group. The random numbers were kept in a sealed location and were opened by an anesthesiologist uninvolved in this study.

\section{Patients}

The inclusion criteria were A) presence of lumbar radiculopathy with a pain intensity $\geq 40 / 100$ in a patient diagnosed with a herniated nucleus pulposus (HNP) or spinal stenosis (SS) after a series of physical, neurologic, and radiologic examinations; $B$ ) one-level TFESI from $L 3$ to L5; C) no prior therapeutic TFESI within the previous 6 months; and D) no prior surgery. The exclusion criteria were A) long-term oral steroid treatment, B) pregnancy, C) cognitive impairment, and D) use of any anti-coagulant.

\section{Outcome Measures}

The primary outcome measure for this study was injectate distribution. To examine the injection distribution pattern, the spread was confirmed using fluoroscopic images (anteroposterior and lateral view), and the maximum distribution was determined. The spreading pattern in each patient was analyzed by a single experienced pain physician and a single experienced radiologist, neither of whom was involved in the procedure. If the 2 physicians did not agree on the results of the analysis, an additional experienced pain physician assessed the fluoroscopic images, and a consensus was reached. The spreading patterns were described as unilateral, bilateral, ventral, or dorsal. Ventral or dorsal flow was also described as being cephalad or caudad, respectively. In the SP group, the flow level was defined as being from the pedicle at the injection site to the cephalad or caudad pedicle (13). Thus, if an injection was performed at the L4 level and the injectates reached the $L 3$ pedicle, the injection would be recorded as one level of cephalad flow. If the injectates reached the $L 5$ pedicle, the injection would be recorded as one level of caudad flow (Fig. 2A). In the RD group, the flow level was defined as being from the IVD at the site of injection to the cephalad or caudad IVD (the superior aspect of the adjacent superior

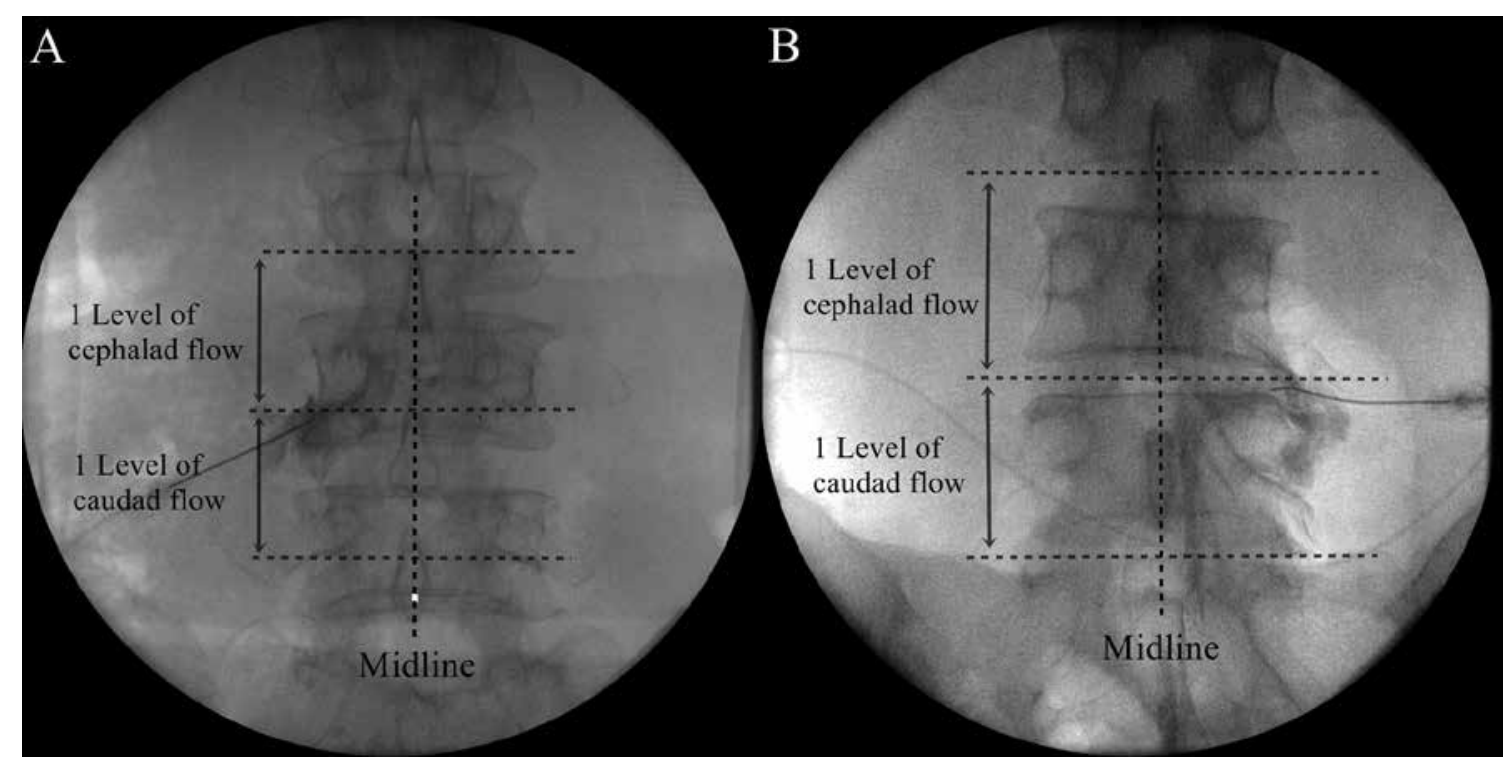

Fig. 3. Levels of epidural spreading. (A) Anteroposterior view of the subpedicular approach at the L4/5 intervertebral level. Note the inferior aspect of the adjacent superior pedicle and the inferior aspect of the adjacent inferior pedicle. (B) Anteroposterior view of the retrodiscal approach at the L4/5 intervertebral level. Note the superior aspect of the adjacent superior $I V D$ and the inferior aspect of the adjacent inferior IVD. 
IVD and the inferior aspect of the adjacent inferior IVD, respectively) (14). Thus, if an injection was performed at the L4/5 IVD and the injectates reached the L3/4 IVD, the injection would be recorded as one level of cephalad flow. If the injectates reached the L5/S1 IVD, the injection would be recorded as one level of caudad flow (Fig. 2B).

The secondary outcome measures were pain relief and reduction of functional disability at 4 weeks after the procedure. Pain relief was assessed using the Visual Analog Scale (VAS, range 0 - 100). Reduction of functional disability was evaluated by the Korean version of the Roland-Morris Disability Questionnaire (RMDQ, range 0 - 24) (15). All complications and side effects were recorded.

\section{Procedures}

All of the TFESls were performed in a procedure suite at an ambulatory surgery center. The first author was present for all procedures performed. We performed TFESI with high-volume injectates $(9 \mathrm{~mL}$ total; $0.33 \%$ lidocaine with $5 \mathrm{mg}$ of dexamethasone mixed with $5 \mathrm{~mL}$ of contrast medium) in both groups. A total of $9 \mathrm{~mL}$ injectate was given in 3 injections $(0.5 \mathrm{~mL}, 2.5$ $\mathrm{mL}$, and $6 \mathrm{~mL}$ ) at 10-second intervals. The injectate was introduced under real-time continuous fluoroscopy at a consistent rate of 0.5 to $1.0 \mathrm{~mL} / \mathrm{sec}$ and monitored manually.

Each patient was placed in the prone position with a pillow under the lower abdomen and above the iliac crest to reduce lumbar lordosis, prepared for the injection, and draped using a sterile technique. The cephalo-caudal angle of the C-arm was adjusted, allowing the incident $x$-ray beam to be parallel to the inferior and superior end plates of the IVD. Vital signs were monitored.

In the SP group, the right and left angles of the C-arm were rotated toward the lesion site by $15-25$ degrees. A 22-gauge, 5-inch spinal needle was guided inferior to the pars interarticularis and into the intervertebral foramen. With the use of biplanar visualization, the needle was advanced into the "safe triangle," which was inferior to the pedicle and superolateral to the exiting spinal nerve. In the lateral projection, needle placement was confirmed with the needle tip approximately 1 to $2 \mathrm{~mm}$ dorsal to the posterolateral vertebral body.

In the RD group, the right and left angles of the C-arm were rotated toward the lesion site by $40-50$ degrees so that the superior articular process was po- sitioned and visible in the middle of the IVD. At this position, a 22-gauge, 5-inch spinal needle was directed to the middle of the IVD, using the lateral aspect of the superior articular process. The needle was advanced until the tip reached the outer wall of the annulus fibrosus. In this process, special care was taken to ensure that the needle did not penetrate the annulus fibrosus of the IVD, with particular attention given to the anteroposterior and the lateral C-arm images.

\section{Statistical Aanalysis}

To calculate the required sample size, data from our previous study were taken into consideration (16). A total of 50 patients were determined to be required to detect a $30 \%$ decrease in the VAS score between the groups with a power of 0.8 and $\alpha$ equal to 0.05 .

The normal distribution of continuous variables was evaluated by the Shapiro-Wilk test. Parametric data were analyzed using the independent $t$-test, while nonparametric data were analyzed with the Mann-Whitney $\mathrm{U}$-test and the Wilcoxon signed-rank test. Descriptive variables were evaluated by the $\chi 2$ test. Continuous variables are expressed as mean \pm standard deviation, whereas ordinal data and nonparametric data are expressed as median (interquartile range; P25 - P75) or number. The degree of agreement among observers was expressed using Kappa values with confidence intervals.

$P$-values $<0.05$ were considered statistically significant. All statistical analyses were performed using SPSS 18 software (Chicago, IL, USA) except for Kappa values, which were obtained using SAS software version 9.1 (SAS Institute, Cary, NC).

\section{Results}

The 50 patients who visited the pain clinic were randomized into equal groups of 25 patients each. In the SP group, 2 patients were lost to follow-up. Consequently, 23 patients remained in the SP group, and 25 were present in the RD group (Fig. 3). There were no significant differences between the 2 groups in demographic data (Table 1).

\section{Primary Outcomes}

All patients demonstrated ventral epidural spread. The total numbers of vertebral segments marked by injectate spread were not significantly different between the 2 groups. However, in $3 \mathrm{~mL}$ of injectate, the injectate spread showed a statistically significantly $(P=0.04)$ stronger bilateral distribution in the RD group (Table 2). 
Epidural Spreading Patterns

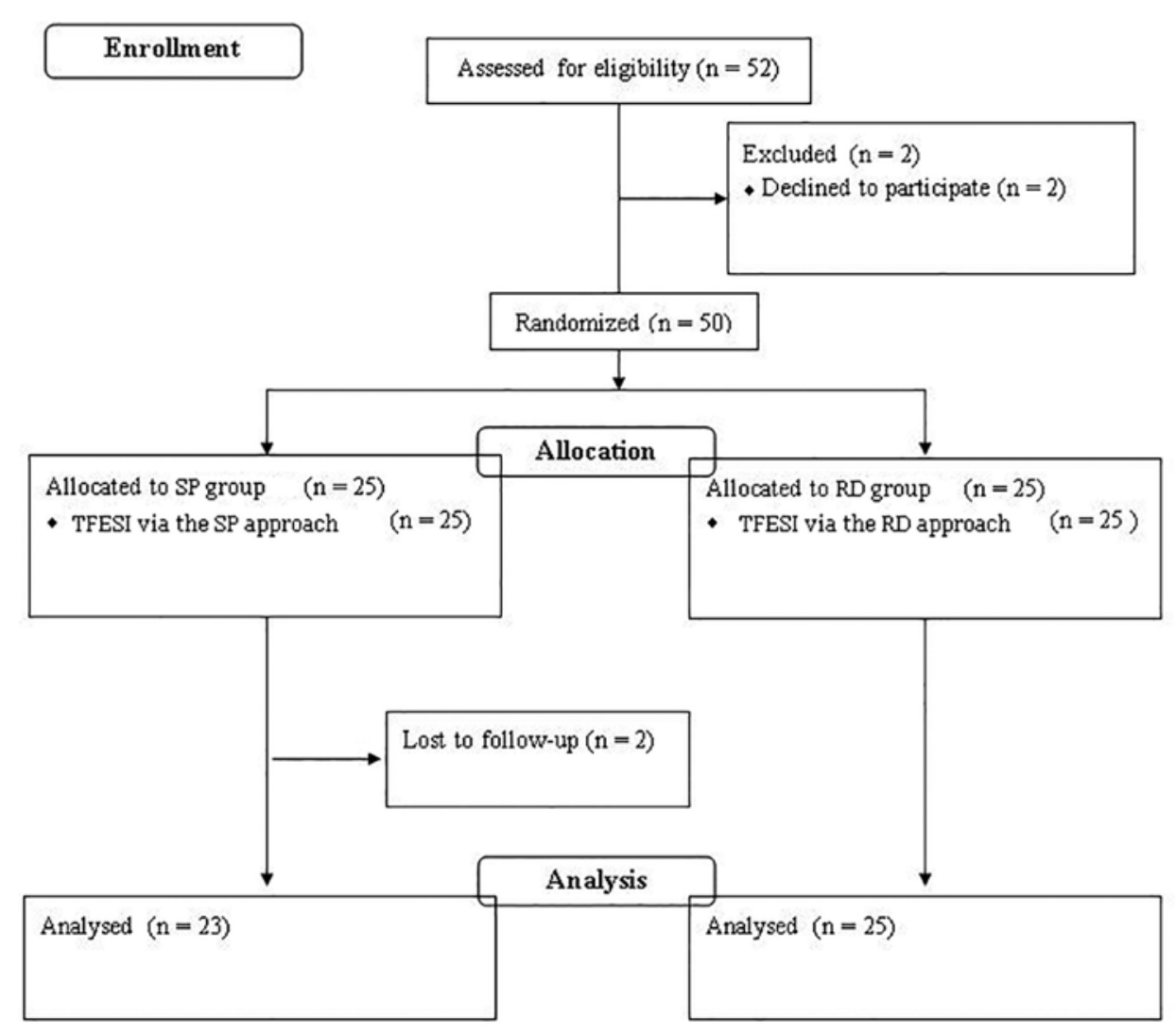

Fig. 3. CONSORT flow diagram.

Table 1. Demographic data

\begin{tabular}{|c|c|c|c|}
\hline & SP Group $(n=23)$ & RD Group $(n=25)$ & $P$-value \\
\hline Age (y) & $60.3 \pm 13.7$ & $57.2 \pm 13.8$ & 0.43 \\
\hline $\operatorname{Sex}(M / F)$ & $8 / 15$ & $14 / 11$ & 0.14 \\
\hline Height $(\mathrm{cm})$ & $160.2 \pm 9.2$ & $163.4 \pm 11.2$ & 0.28 \\
\hline Weight (kg) & $62.3 \pm 10.6$ & $63.6 \pm 11.3$ & 0.68 \\
\hline Levels injected (L3/L4/L5) & $3 / 13 / 7$ & $1 / 21 / 3$ & 0.11 \\
\hline Injection site (Left / Right ) & $11 / 12$ & $12 / 13$ & 0.99 \\
\hline \multicolumn{4}{|l|}{ Radiologic findings } \\
\hline Central stenosis & 10 & 12 & 0.75 \\
\hline Foraminal stenosis & 9 & 6 & 0.26 \\
\hline L3/4 HNP & 8 & 3 & 0.06 \\
\hline L4/5 HNP & 14 & 16 & 0.82 \\
\hline L5/S1 HNP & 4 & 3 & 0.59 \\
\hline
\end{tabular}

Data are expressed as mean \pm standard deviation or number.

HNP: herniated nucleus pulposus 
Table 2. Epidural spreading patterns

\begin{tabular}{|c|c|c|c|c|}
\hline & & $\begin{array}{c}\text { SP Group } \\
(n=23)\end{array}$ & $\begin{array}{c}\text { RD Group } \\
(\mathrm{n}=\mathbf{2 5})\end{array}$ & $P$-value \\
\hline \multicolumn{5}{|c|}{ Number of patients } \\
\hline \multirow[t]{5}{*}{$0.5 \mathrm{~mL}$} & Unilateral / Bilateral & $23 / 0$ & $25 / 0$ & $>0.99$ \\
\hline & Level of ventral cephalad flow $(0 / 1 / 2 / 3)$ & $23 / 0 / 0 / 0$ & $22 / 3 / 0 / 0$ & 0.08 \\
\hline & Level of ventral caudad flow $(0 / 1 / 2 / 3)$ & $23 / 0 / 0 / 0$ & $25 / 0 / 0 / 0$ & $>0.99$ \\
\hline & Level of dorsal cephalad flow $(0 / 1 / 2 / 3)$ & $23 / 0 / 0 / 0$ & $25 / 0 / 0 / 0$ & $>0.99$ \\
\hline & Level of dorsal caudad flow $(0 / 1 / 2 / 3)$ & $23 / 0 / 0 / 0$ & $24 / 1 / 0 / 0$ & 0.33 \\
\hline \multirow[t]{5}{*}{$2.5 \mathrm{~mL}$} & Unilateral / Bilateral & $21 / 2$ & $17 / 8$ & 0.04 \\
\hline & Level of ventral cephalad flow $(0 / 1 / 2 / 3)$ & $5 / 14 / 3 / 1$ & $5 / 14 / 6 / 0$ & 0.58 \\
\hline & Level of ventral caudad flow $(0 / 1 / 2 / 3)$ & $14 / 9 / 0 / 0$ & $12 / 13 / 0 / 0$ & 0.37 \\
\hline & Level of dorsal cephalad flow $(0 / 1 / 2 / 3)$ & $12 / 9 / 1 / 1$ & $14 / 11 / 0 / 0$ & 0.51 \\
\hline & Level of dorsal caudad flow $(0 / 1 / 2 / 3)$ & $12 / 11 / 0 / 0$ & $18 / 7 / 0 / 0$ & 0.16 \\
\hline \multirow[t]{5}{*}{$6 \mathrm{~mL}$} & Unilateral / Bilateral & $11 / 12$ & $11 / 14$ & 0.79 \\
\hline & Level of ventral cephalad flow $(0 / 1 / 2 / 3)$ & $4 / 6 / 6 / 7$ & $1 / 6 / 10 / 8$ & 0.59 \\
\hline & Level of ventral caudad flow $(0 / 1 / 2 / 3)$ & $5 / 16 / 2 / 0$ & $8 / 12 / 5 / 0$ & 0.29 \\
\hline & Level of dorsal cephalad flow $(0 / 1 / 2 / 3)$ & $7 / 7 / 3 / 6$ & $8 / 5 / 8 / 4$ & 0.39 \\
\hline & Level of dorsal caudad flow $(0 / 1 / 2 / 3)$ & $5 / 15 / 3 / 0$ & $11 / 10 / 4 / 0$ & 0.19 \\
\hline \multicolumn{5}{|c|}{ Median levels of contrast spread from injection site } \\
\hline \multirow[t]{4}{*}{$0.5 \mathrm{~mL}$} & Ventral cephalad flow & $0(0)$ & $0(0)$ & 0.09 \\
\hline & Ventral caudad flow & $0(0)$ & $0(0)$ & $>0.99$ \\
\hline & Dorsal cephalad flow & $0(0)$ & $0(0)$ & $>0.99$ \\
\hline & Dorsal caudad flow & $0(0)$ & $0(0)$ & 0.34 \\
\hline \multirow[t]{4}{*}{$2.5 \mathrm{~mL}$} & Ventral cephalad flow & $1(0)$ & $1(0.5)$ & 0.71 \\
\hline & Ventral caudad flow & $0(1.0)$ & $1(1.0)$ & 0.38 \\
\hline & Dorsal cephalad flow & $0(1.0)$ & $0(1.0)$ & 0.60 \\
\hline & Dorsal caudad flow & $0(1.0)$ & $0(1.0)$ & 0.16 \\
\hline \multirow[t]{4}{*}{$6 \mathrm{~mL}$} & Ventral cephalad flow & $2(2.0)$ & $2(2.0)$ & 0.37 \\
\hline & Ventral caudad flow & $1(0)$ & $1(1.0)$ & 0.97 \\
\hline & Dorsal cephalad flow & $1(3.0)$ & $1(2.0)$ & 0.95 \\
\hline & Dorsal caudad flow & $1(2.0)$ & $1(1.0)$ & 0.26 \\
\hline
\end{tabular}

Data are expressed as median (interquartile range; P25 - P75) or number.

The Kappa value range ( $95 \%$ confidence interval) was $0.7895(0.3903-1)-1$.

\section{Secondary Outcomes}

There were no significant differences in baseline VAS score for back and radicular pain or in RMDQ score between the 2 groups. At 4 weeks after treatment, both groups demonstrated statistically significant improvement in back and leg pain according to VAS score and improvement in functional status according to RMDQ score $(P<0.05)$. No significant differences were observed between the 2 groups (Fig. 4).

No severe adverse events were reported in this study. Specifically, there were no observed dural punctures in either group, no subdural or intrathecal injections, and no intradiscal injections. No patient in either group sustained any infectious complications, postdural puncture cephalalgia, persistent paresthesias, systemic steroid reactions, skin lesions, or any adverse reaction to contrast media or adjuvant medications. However, in the SP group, 2 patients complained of moderate pain on injection, which was resolved immediately after the injection. 


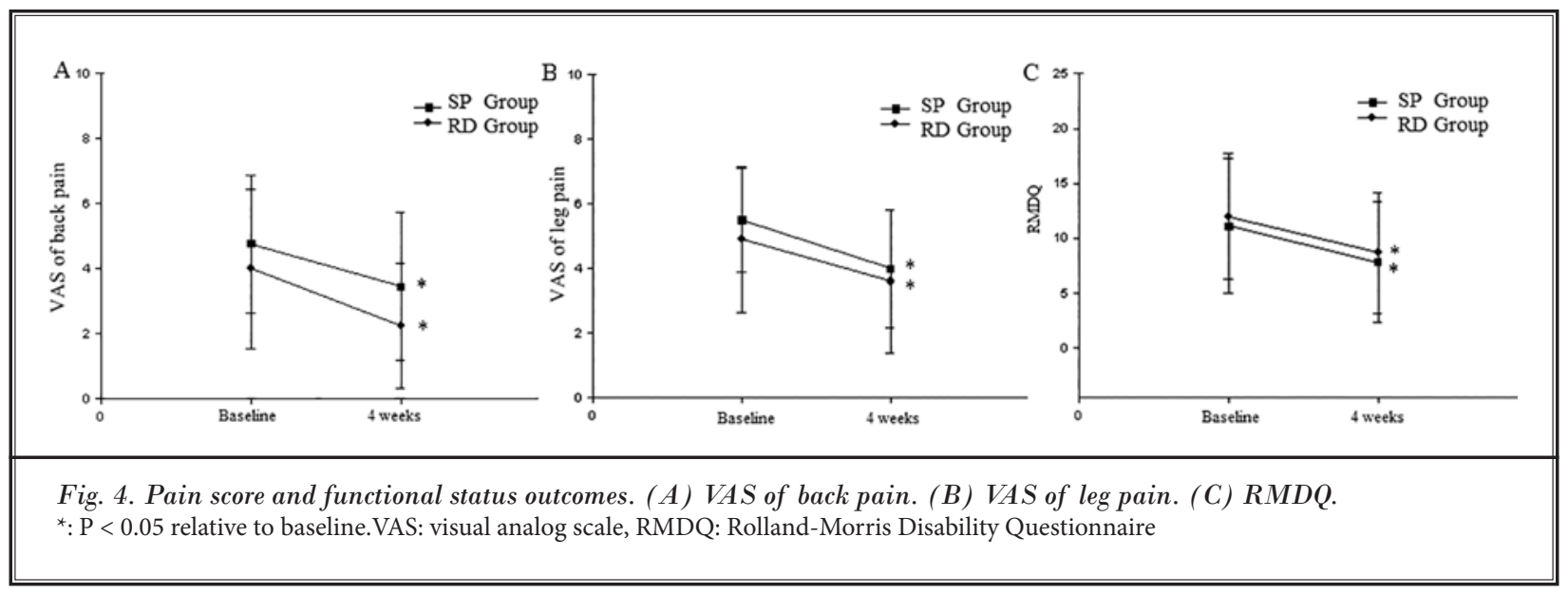

\section{Discussion}

Our results showed that the epidural spreading patterns and clinical outcomes of patients undergoing lumbar TFESIs with high-volume injectate were not significantly different between the SP approach and the RD approach, except that the medication exhibited more extensive bilateral distribution in the RD group in $3 \mathrm{~mL}$ of injectate.

The effect in TFESI appears to be influenced by the volume of the injected material. Rabinovitch et al (7) reported a positive correlation between fluid injection volume into the epidural space and relief of radicular pain. It was suggested that epidural adhesiolysis washes out inflammatory cytokines from the affected area. Furthermore, the increased volume enables lavage of the epidural space, suppression of ectopic discharge from the injured nerve, and enhancement of blood flow to the ischemic nerve roots (16). In this respect, the injectate volume could have a greater impact on TFESI efficacy than the injectate contents. We therefore decided that an injectate volume of $9 \mathrm{~mL}$ would be optimal for epidural adhesiolysis.

The influence of volume on epidural pressure remains controversial. Cardoso et al (17) reported a direct correlation between the volume of anesthetic injected and epidural pressure and Thomas et al (18) also observed higher epidural pressure when larger volumes of local anesthetic were used. In contrast, Paul et al (19) found similar epidural pressures with different injection volumes. Therefore, the decision of when to incorporate high-volume TFESI in patient care should be made at the physician's discretion.

Ensuring good runoff during injection is important to prevent volume-related increase in local pres- sure. When epidural pressure is increased by injection of fluid, the dura must have been compressed. Dural compression could increase CSF $\{\mathrm{sp}\}$ pressure and CSF absorption. Accordingly, an increase in epidural pressure is transmitted directly to the cerebrospinal fluid, and this in turn will cause a rise in intracranial pressure (20). Moreover, an increase in epidural pressure likely contributes to interference with the blood supply of the spinal cord, causing spinal cord infarction $(21,22)$. A preponderance of patients with this complication are elderly male patients with cardiovascular disease and/or cancer $(21,22)$. Therefore, caution should be exercised in patient selection when performing high-volume TFESI.

Botwin et al (13) analyzed epidurograms after TFESI using the SP approach with a low-volume contrast medium $(2 \mathrm{~mL})$. They found that contrast flow extended a mean of 1.13 and 0.6 levels cephalad and caudad from the injection site, respectively. There were no significant differences in contrast flow noted between patients with HNP versus patients with $\mathrm{SS}$. In $3 \mathrm{~mL}$ in the SP group, we found that the contrast flow extended a mean of 1.0 and 0.39 levels cephalad and caudad from the injection site, respectively (not shown in Table 2). Moreover, there was no difference in injectate distribution between the 2 groups, regardless of HNP or SS.

Kim et al (23) compared the patterns of contrast spread between the SP and RD approach with lowvolume injectates (1 $\mathrm{mL}$ of nonionic contrast medium) in patients with $L 5$ radiculopathy. They reported that the contrast medium diffused around the L5 and S1 nerve roots in $67 \%$ of all patients for whom the RD approach was used, whereas the medium diffused around only the $L 5$ root in $73 \%$ of all patients for whom the SP 
approach was used. However, our results showed that the caudad spread was not statistically significantly different between the 2 groups, for either a $0.5 \mathrm{~mL}$ or 3 $\mathrm{mL}$ injectate volume. Our results showed that $3 \mathrm{~mL}$ injectates spread significantly bilaterally in the RD group, which suggests that the RD approach might be more useful when low-volume injectates are used in patients with bilateral symptoms.

Anatomical diversity might explain the slight differences between our results and previous results. The unpredictable spread pattern is likely due to anatomical variation of the epidural space and the complex structure of the epidural space (24). Significant interindividual variation was observed with respect to both area and spread pattern. It is not possible to predict whether the anesthetic will have a cephalad or caudal spread, spread unilaterally, or show a general spread pattern. Burn et al (25) reported that it is impossible to accurately predict the level attained after epidural injection of contrast medium.

The anterior epidural space is isolated from the rest of the epidural space and is nearly filled with veins except for the area caudal to the L4-L5 disk, where a fat-filled anterior epidural space develops (26). Hogan evaluated cryomicrotome sections of the anterior epidural space and found that the anterior space appears to be filled with veins (26) that rarely cross the midplane of the posterior longitudinal ligament (PLL) or its lateral membrane $(27,28)$. The epidural space is filled by a thin layer of areolar connective tissue termed the epidural membrane (29). An anterior midline septum has been identified that divides the anterior compartment (30). Hogan et al (31) also observed a midline tongue of connective tissue extending from the IVD, reaching caudad and to a lesser degree cephalad in the PLL and posterior vertebral body.

Increasing the injectate volume did not result in a linear increase in cervical epidural spread (23). The number of cervical vertebral segments in which $1 \mathrm{~mL}$ of epidural medication is distributed is likely to decrease as the injectate volume increases. Regardless of the injected contrast medium volume and the procedure level, epidurography showed more extensive distribution in the cephalad direction compared to the caudad direction (32). These findings are consistent with our results. Moreover, a large-volume injection can clearly show circumferential contrast spread; for instance, epidurograms with $10 \mathrm{~mL}$ contrast medium are more clearly opacified than those with $5 \mathrm{~mL}$ (33). We found a higher incidence of dorsal spread with $9 \mathrm{~mL}$ injectates than with 0.5 or $3 \mathrm{~mL}$ injectates, a finding that agrees with previous results that circumferential spread is proportional to volume.

When TFESI is performed, the contrast medium must spread over the proximal region along the anterior lateral part of a specific nerve (34). However, a considerable amount of the drug injected via the SP approach sometimes flows into the distal region along the spinal nerve. This makes it difficult to insert the injection needle in cases of severe SS, when the needle is supposed to be located at the region more anterior to the intratransforaminal epidural nerve root (23). Lew et al (35) have suggested that, because a rostral spread of injectate to bathe the preganglionic portion of the nerve root cannot always be guaranteed, an RD approach at the level of the supra-adjacent IVD could be helpful. Theoretically, by utilizing the RD approach for TFESI, the injectate can be placed closer to the site of neural impingement, thereby providing a more effective washout of inflammatory disc material $(35,36)$. However, a systematic review and meta-analysis of TFESIs with the SP and RD approaches reported that the VAS scores of the 2 techniques were not statistically significantly different (37). Our results also showed similar pain reduction between the 2 groups.

Using the RD approach, the needle tip may unintentionally be placed too far ventrally and enter the IVD. Levi et al (38) performed 257 RD TF injections, and demonstrated a $4.7 \%$ rate of intradiscal injection. Needle entry into the IVD may be of concern for several reasons. First, infectious discitis, which is a rare occurrence following provocation discography (39). Second, there may be an associated increased risk of future disc degeneration or herniation induced by needle puncture (40). Therefore, decisions regarding use of the RD approach should take into account discogram.

Although there were no serious complications in our study, 2 patients in the SP group experienced temporary severe pain in the lower back and lower extremities during drug injection. This complication was expected, since the drug was injected into a spinal canal that was likely narrowed due to HNP, SS, or epidural fibrosis (41).

This study had several limitations. First, we enrolled only a small number of patients, and the study was not powered to find small differences in spreading patterns or pain relief between the 2 groups. Second, slightly different methods were used in the 2 groups to determine the number of levels at which the injectate was spread. Third, patients with severe SS or HNP were included, 
and the injected drugs spread to the distal nerve root in some cases. Fourth, we did not analyze long-term outcomes and focused only on the VAS and RMDQ.

\section{Conclusion}

In conclusion, epidural spreading patterns and clinical outcomes, including pain relief and reduced functional disability, were not significantly different between the SP approach and the RD approach in patients undergoing lumbar TFESI with a high-volume injectate. Moreover, in TFESI with low-volume injectates, the RD approach might be more beneficial for patients with bilateral symptoms.

\section{Acknowledgments}

Author Contributions: Dr. PHS and KWJ had full access to all the data in the study and takes responsibility for the integrity of the data and the accuracy of the data analysis. Dr. PHS designed the study protocol. Dr. $\mathrm{PHS}, \mathrm{YSH}$ and KWJ managed the literature searches and summaries of previous related work and wrote the first draft of the manuscript. Dr. PHS, KWJ, SHY provided revision for intellectual content and final approval of the manuscript.

\section{Conflict of Interest}

All authors have no conflicts of interest to report. None of the authors of the manuscript received any remuneration. Further, the authors have not received any reimbursement or honorarium in any other manner. The authors are not affiliated in any manner. All the authors are members of the Korean Society of Anesthesiologists and practicing interventional pain physicians.

\section{Funding/Support}

The authors wish to disclose and thank the sponsor of the study. This study was supported by intramural research promotion grants from Ewha Womans University School of Medicine (Level 3). The sponsorship was limited to supplies and expenses. The sponsorship included payment for data entry, and analysis of the data. They had no influence or interference after the protocol was designed.

\section{Role of Sponsor}

The financial sponsor of this work had no role in the design and conduct of the study or the collection, management, analysis, and interpretation of the data. The sponsor also did not have a role in the preparation or review of the manuscript or the decision to submit.

\section{Information on author access to data}

The datasets used and/or analyzed during the current study are available from the corresponding author on reasonable request.

We would like to thank the editorial board of Pain Physician for review and criticism in improving the manuscript.

\section{References}

11. Vad VB, Bhat AL, Lutz GE, Cammisa F. Transforaminal epidural steroid injections in lumbosacral radiculopathy: A prospective randomized study. Spine 2002; 27:11-16.

2. Kim LK, Kim JR, Shin SS, Kim IJ, Kim BN, Hwang GT. Analysis of influencing factors to depth of epidural space for lumbar transforaminal epidural block in korean. Korean J Pain 2011; 24:216-220.

3. Manchikanti L, Buenaventura RM, Manchikanti KN, Ruan X, Gupta S,Smith HS, Christo PJ, Ward SP. Effectiveness of therapeutic lumbar transforaminal epidural steroid injections in managing lumbar spinal pain. Pain Physician 2012; 15:E199-E245.
4. Benny B, Azari P. The efficacy of lumbosacral transforaminal epidural steroid injections: A comprehensive literature review. J Muscul Rehabil 2011; 24:67-76.

5. Wilkinson IM, Cohen SP. Epidural steroid injections. Curr Pain Headache Rep 2012; 16:50-59.

6. Wilkinson I, Cohen SP. Epidural steroids for spinal pain and radiculopathy: A narrative, evidence-based review. Curr Opin Anaesthesiol 2013; 26:562-572.

7. Rabinovitch DL, Peliowski A, Furlan AD. Influence of lumbar epidural injection volume on pain relief for radicular leg pain and/or low back pain. Spine 2009; 9:509-517.

8. Bogduk N, Endres SM. Clinical anatomy of the lumbar spine and sacrum 4th edition. Elsevier/Churchill Livingstone, New York, 2005.

9. Bogduk N. Practice Guidelines Spinal Diagnostic Treatment and Procedures First Ed. International Spine Intervention Society, San Francisco, 2004, pp. 177.

10. Jasper JF. Lumbar retrodiscal transforaminal injection. Pain Physician 2007; 10:501-510.

11. Jeong HS, Lee JW, Kim SH, Myung JS, Kim JH, Kang HS. Effectiveness of transforaminal epidural steroid injection by using a preganglionic approach: A prospective randomized controlled study. Radiology 2007; 245:584-590. 
12. Park CH, Lee SH, Park HS. Lumbar retrodiscal versus post-ganglionic transforaminal epidural steroid injection for the treatment of lumbar intervertebral disc herniations. Pain Physician 2011; 14:353-36o.

13. Botwin K, Natalicchio J, Brown LA. Epidurography contrast patterns with fluoroscopic guided lumbar transforaminal epidural injections: A prospective evaluation. Pain Physician 2004; 7:211-215.

14. Furman MB, Mehta AR, Kim RE, Simon Jl, Patel R, Lee TS, Reeves RS. Injectate volumes needed to reach specific landmarks in lumbar transforaminal epidural injections. PM \& $R$ 2010; 2:625-635.

15. Lee JS, Lee DH, Suh KT, Kim JI, Lim JM, Goh TS. Validation of the Korean version of the Roland-Morris Disability Questionnaire. Eur Spine ] 2011; 20:2115-2119.

16. Chun EH, Park HS. Effect of high-volume injectate in lumbar transforaminal epidural steroid injections: A randomized, active control trial. Pain Physician 2015; 18:519-525.

17. Cardoso MM, Carvalho JC. Epidural pressures and spread of $2 \%$ lidocaine in the epidural space: Influence of volume and speed of injection of the local anesthetic solution. Reg Anesth Pain Med 1998; 23:14-19.

18. Thomas PS, Gerson JI, Strong G. Analysis of human epidural pressures. Reg Anesth 1992; 17:212-215.

19. Paul DL, Wildsmith JA. Extradural pressure following the injection of two volumes of bupivacaine. $\mathrm{Br}$ J Anaesth 1989; 62:368-372.

20. Usubiaga JE, Wikinski JA, Usubiaga LE. Epidural pressure and its relation to spread of anesthetic solutions in epidural space. Anesth Analg 1967; 46:440-446.

21. Skouen JS, Wainapel SF, Willock MM. Paraplegia following epidural anesthesia. A case report and a literature review. Acta Neurologica Scandinavica 1985; 72:437-443.

22. Bhuiyan MS, Mallick A, Parsloe M. Postthoracotomy paraplegia coincident with epidural anaesthesia. Anaesthesia 1998; 53:583-586.

23. Kim C, Moon CJ, Choi HE, Park Y. Retrodiscal approach of lumbar epi- dural block. Annals Rehabil Med 2011; 35:418-426.

24. Savolaine ER, Pandya JB, Greenblatt SH, Conover SR. Anatomy of the human lumbar epidural space: New insights using CT-epidurography. Anesthesiology 1988; 68:217-220.

25. Burn JM, Guyer PB, Langdon L. The spread of solutions injected into the epidural space. A study using epidurograms in patients with the lumbosciatic syndrome. Br J Anaesth 1973; 45:338-345.

26. Hogan $\mathrm{QH}$. Lumbar epidural anatomy. A new look by cryomicrotome section. Anesthesiology 1991; 75:767-775.

27. Gershater R, St Louis EL. Lumbar epidural venography. Review of 1,200 cases. Radiology 1979; 131:409-421.

28. Meijenhorst GC. Computed tomography of the lumbar epidural veins. Radiology 1982; 145:687-691.

29. Wiltse LL, Fonseca AS, Amster J, Dimartino P, Ravessoud FA. Relationship of the dura, Hofmann's ligaments, Batson's plexus, and a fibrovascular membrane lying on the posterior surface of the vertebral bodies and attaching to the deep layer of the posterior longitudinal ligament. An anatomical, radiologic, and clinical study. Spine 1993; 18:1030-1043.

30. Schellinger D, Manz HJ, Vidic B, Patronas NJ, Deveikis JP, Muraki AS,Abdullah DC. Disk fragment migration. Radiology 1990; 175:831-836.

31. Hogan QH. Epidural anatomy examined by cryomicrotome section. Influence of age, vertebral level, and disease. Reg Anesth 1996; 21:395-406.

32. Pfirrmann CW, Oberholzer PA, Zanetti M, Boos N, Trudell DJ, Resnick D, Hodler J. Selective nerve root blocks for the treatment of sciatica: Evaluation of injection site and effectiveness--a study with patients and cadavers. Radiology 2001; 221:704-711.

33. Yokoyama M, Hanazaki M, Fujii $H$, Mizobuchi S, Nakatsuka H, Takahashi T, Matsumi M, Takeuchi M, Morita K. Correlation between the distribution of contrast medium and the extent of blockade during epidural anesthesia. Anesthesiology 2004; 100:1504-1510.

34. Smuck M, Fuller BJ. Author reply to
"Letter to the editor" by Manchikanti and Singh (Spine J. 2007(5)) regarding "Incidence of simultaneous epidural and vascular injection during lumbosacral transforaminal epidural injections". The spine journal : official journal of the North American Spine Society 2007; 7:749.

35. Lew HL, Coelho P, Chou LH. Preganglionic approach to transforaminal epidural steroid injections. American Journal of Physical Medicine \& Rehabilitation 2004: 83:378.

36. Lee JW, Kim SH, Choi JY, Yeom JS, Kim KJ, Chung SK, Kim HJ, Kim C, Kwack KS, Kwon JW, Moon SG, Jun WS, Kang HS. Transforaminal epidural steroid injection for lumbosacral radiculopathy: Preganglionic versus conventional approach. Korean Journal of Radiology 2006; 7:139-144.

37. Pairuchvej S, Arirachakaran A, Keorochana G, Wattanapaiboon K,Atiprayoon $S$, Phatthanathitikarn P, Kongtharvonskul J. The short and midterm outcomes of lumbar transforaminal epidural injection with preganglionic and postganglionic approach in lumbosacral radiculopathy: A systematic review and meta-analysis. Neurosurgical Review 2017.

38. Levi D, Horn S, Corcoran S. The incidence of intradiscal, intrathecal, and intravascular flow during the performance of retrodiscal (infraneural) approach for lumbar transforaminal epidural steroid injections. Pain Med 2016; 17:1416-1422.

39. Osti OL, Fraser RD, Vernon-Roberts B. Discitis after discography. The role of prophylactic antibiotics.] Bone Joint Surg British volume 1990; 72:271-274.

40. Carragee EJ, Don AS, Hurwitz EL, Cuellar JM, Carrino JA, Herzog R. 2009 ISSLS Prize Winner: Does discography cause accelerated progression of degeneration changes in the lumbar disc: A tenyear matched cohort study. Spine 2009; 34:2338-2345.

41. Botwin KP, Gruber RD, Bouchlas CG, Torres-Ramos FM, Freeman TL, Slaten WK. Complications of fluoroscopically guided transforaminal lumbar epidural injections. Arch Phys Med Rehabil 2000; 81:1045-1050. 